\title{
Computergestützte AnAlyse UND HIT-SONGWRITING
}

\section{Frank Riedemann}

Kommerziell erfolgreiche Popmusik ${ }^{1}$ weist formatspezifische Strukturen und Konventionen auf, die im engen Zusammenhang mit der Chartkompatibilität eines Songs stehen. So betonen professionelle Songwriter immer wieder, dass erst die intensive analytische Auseinandersetzung mit bestehenden Hitsongs die Basis für erfolgreiches Songwriting schafft (z. B. Silver/Bruce 1939; Tucker 2003; Chambers 2005). Im Zuge des Analyseprozesses werden musikund textimmanente Strukturen erkannt und internalisiert, wodurch ein Repertoire an alternativen Ausdrucks- und Gestaltungsmitteln aufgebaut wird. ${ }^{2}$ Vor diesem Hintergrund ist es nicht weiter verwunderlich, dass ca. 90 Prozent der erfolgreichsten Hits aus den aktuellen deutschen Singlecharts exklusiv oder durch Co-writing aus der Feder professioneller Songwriter stammen (vgl. Riedemann, in Vorb.). Dennoch stehen viele Musiker einer analytisch-theoretischen Reflektion im Bereich Songwriting skeptisch gegenüber, da dies dem medial propagierten Ideal des naiv-unmittelbaren Schöpfungsakts als Quasi-Beweis für die Authentizität eines Künstlers widerspricht. Dabei ließen sich durch eine profunde Analyse potentielle Inkompatibilitäten mit dem avisierten Format vermeiden: »People listen to songs on the radio, then do the opposite. The rules are there - why do so few composers bother to learn them?« (Curtis Mayfield, zit. n. Hirschhorn 2001: xxiv). Derartige »Regelverstöße« im Sinne einer Abweichung von formatspezifischen Konventionen lassen sich nicht nur im Amateurbereich, sondern auch bei Non-Hits im professionellen Kontext entdecken wie ein Vergleich mit Hitsongs nachfolgend zeigen wird. Hierbei soll diskutiert werden,

1 Die Bezeichnung »Popmusik« wird hier als Oberbegriff für populären Mainstream gebraucht, wie man inn insbesondere in den Charts vorfindet. Zur definitorischen Problematik populärer Musikformen und des Genrebegriffs sei hier auf die einschlägige Literatur verwiesen: z. B. Middleton 2001; Rösing 2005; die Problematik der Genrezuweisung wird ausführlich bei Fabbri 2004 diskutiert.

2 Kachulis (2003: ix) verwendet in diesem Zusammenhang den Begriff »internal library«. 
welchen Beitrag die Musikwissenschaft durch computergestützte Analyseverfahren zur Professionalisierung und Erweiterung der kreativen Ausdrucksmöglichkeiten im Bereich Hitsongwriting leisten kann.

Zunächst ein kurzer Exkurs zur Ausbildungssituation. Das Berklee College of Music in Boston ist laut eigenem Bekunden die weltweit einzige akademische Institution, die Songwriting als Hauptfach anbietet (Kachulis 2010). Darüber hinaus wird Songwriting als Teildisziplin im Rahmen der praktischen Popmusikerausbildung unterrichtet, wie z.B. am Liverpool Institute for Performing Arts, an der Popakademie in Mannheim oder im Rahmen des Kontaktstudiengangs Popularmusik in Hamburg. In Ermangelung weiterführender Standardliteratur sind die Studieninhalte hierbei hochgradig personalisiert und reflektieren die Erfahrungswerte der jeweiligen Dozenten, die sich z.T. gleichzeitig als Autoren für die einführende unterrichtsbegleitende Literatur verantwortlich zeichnen (u.a. Pattison 1991; Perricone 2000; Kachulis 2003; Abou-Dakn 2006). Überdies richtet sich die gesamte Publikationsbreite zum Thema Songwriting immer auch an den musiktheoretischen Laien, sodass auf die Darstellung komplexer musikalischer Sachverhalte weitestgehend verzichtet wird (ein Überblick zur Songwritingliteratur findet sich bei Schmidt/ Terhag 2010: 102f.). Zusammenfassend kann festgehalten werden, dass im Gegensatz zum klassischen Kompositionsstudium weder Ausbildungsstandards für den Fachbereich Songwriting existieren noch weiterführende Literatur verfügbar ist. Im Anbetracht der Tatsache, dass in der Songwritingliteratur sehr unterschiedliche Ausdrucks- und Gestaltungsmerkmale als hitkonstituierend bezeichnet werden, stellt sich die Frage, inwiefern diese Features in der von den Autoren proklamierten Frequenz in Hitsongs tatsächlich statistisch nachweisbar sind. Hierzu ein Blick auf den aktuellen Forschungsstand in der Musikwissenschaft:

Die Forschungslage im Bereich der Analyse populärer Musik ist nach wie vor geprägt durch den Einfluss der Cultural Studies, die kulturelle Aneignungs- und Rezeptionskontexte in den Vordergrund stellen. Dennoch lässt sich in den letzten Jahren ein Trend zur Rückbesinnung auf die Analyse der klingenden Musik als eine der traditionellen Kernkompetenzen der Musikwissenschaft beobachten. In diesem Zusammenhang steht immer noch die Frage nach einem angemessenen methodischen Zugang aus, was sich unter anderem in einer großen Methodenvielfalt niederschlägt (vgl. Middleton 2000; Moore 2003). Im Bereich musikanalytisch ausgerichteter Studien dominieren in erster Linie hermeneutische Einzel- oder Werkanalysen mit Fokus auf einen einzelnen Künstler oder einer Gruppe (Elflein 2010: 11). Obgleich die Volksmusikforschung bereits auf eine lange Tradition rechnergestützter 
statistisch-analytischer Studien verweisen kann (z. B. Steinbeck 1982; Jesser 1991; Eerola et al. 2001), liegen nur sehr wenige Forschungsergebnisse über die statistische Verteilung von musik- und textimmanenten Merkmalen im Bereich aktueller populärer Musik vor (vgl. Kopiez/Müllensiefen 2011). Verantwortlich für dieses Forschungsdefizit sind nicht zuletzt forschungsökonomische Gründe: Es gibt aufgrund des geltenden Urheberrechts keine frei verfügbaren Datenbanken aktueller populärer Musik, die in einem analysefähigem computerlesbaren Format vorliegen. So steht zu Beginn der Forschung stets eine sehr aufwendige Transkription und Kodierung der Songs. Für den Fall, dass die existierenden Softwarepakete nicht die benötigten Analysefunktionen aufweisen, muss überdies eine Einarbeitung in die entsprechende Befehlssyntax des jeweiligen Analysetools oder aber die enorm zeitintensive Programmierung einer neuen Analysesoftware erfolgen. Beides verlangt profunde Programmierkenntnisse, die in den seltensten Fällen bei einem Musikwissenschaftler vorausgesetzt werden können.

Die Ergebnisse des nachfolgenden Vergleichs zwischen Hits und Non-Hits sind im Kontext einer Forschungsarbeit zur computergestützten Analyse musik- und textimmanenter Gestaltungsmerkmale aktueller Popmusik entstanden (Riedemann, in Vorb.). Im weiteren Verlauf sollen exemplarisch sowohl ausgewählte Rahmenvariablen vorgestellt werden, die einen bestimmten Wertebereich innerhalb des vorliegenden Hitformats beschreiben, als auch Variablen, die auf komplexe kombinatorische Realisierungen von musik- und textimmanenten Strukturen verweisen. Die Auswahl der Variablen ist das Ergebnis einer intensiven Auseinandersetzung mit dem Material und basiert sowohl auf musiktheoretischen und -psychologischen Überlegungen als auch auf Erfahrungen des Autors als professionellem Songwriter. ${ }^{3}$ Nachfolgend werden aus Gründen der Übersichtlichkeit nur Merkmale vorgestellt, die den Chorus als Zentralsektion eines Songs betreffen. ${ }^{4}$ Im Anschluss wird eine binär-logistische Regression das Potential der computergestützten Analyse als auch die Beschränkungen eines hohen Abstraktionsniveaus verdeutlichen.

Die zu analysierenden Songs wurden auf Grundlage des Notentextes in ein computerlesbares Format kodiert und in die vom Autor entwickelte Analysesoftware Essencer eingegeben. Das Programm stellt mit mehr als 1.600

3 Der Hinweis auf die Tätigkeit des Autors als Songwriter ist insofern bedeutsam, als dass der individuelle Standpunkt des Analysierenden zwangsläufig einen Einfluss auf den Analyseprozess ausübt, vgl. Redmann 2002: 203ff.

4 Der Begriff Zentralsektion wird insbesondere im Umfeld des Berklee College of Music gebraucht. Er trägt dem Umstand Rechnung, dass hier die zentrale textliche Idee bzw. der Hook eines Songs zu finden ist, vgl. Pattison 1991: 53. 
Seiten Programmcode eine Vielzahl von Funktionen zur Verfügung, die speziell auf die Anforderungen der Popmusikanalyse zugeschnitten sind. Obgleich der Notentext die erklingende Musik nur bedingt in ihrer »sonischen Wirklichkeit« (Wicke 2003: 123) und den damit verbundenen subjektiven Erlebnisqualitäten repräsentiert, bildet dieser dennoch wichtige musikstrukturelle Bezüge $a b$, wie neuere musikpsychologische Forschungen bestätigen (Huron 2006; Müllensiefen et al. 2008). Dennoch soll hier mitnichten der Eindruck eines Primats des Notentextes als Analysegrundlage erweckt werden - die Komplexitätsreduktion ist in erster Linie forschungspragmatischen Überlegungen geschuldet (zur Problematik der »notational centricity« vgl. Middleton 2000: 4).

Als Hitsongs wurden 57 der erfolgreichsten Titel aus den deutschen Singlejahrescharts der Jahre 2000-2005 ausgewählt, wobei jedes Jahr mit mindestens neun Songs vertreten ist. ${ }^{5}$ Als Non-Hits wurden jene Songs klassifiziert, die zusammen mit einem der zuvor genannten Hits auf einem Album erschienen sind, aber keine Chartnotierung als Single aufweisen. Obgleich diese Arbeitsdefinition von Non-Hits als eine stark verkürzte Annäherung an ein äußerst vielschichtiges Phänomen zu bewerten ist, stellt sie dennoch im Kontext der nachfolgenden Untersuchung eine hinreichend praktikable Lösung für die Auswahl des Vergleichskorpus dar. Die Auswahl der Non-Hits erfolgte durch eine Zufallsfunktion mit der Vorgabe, dass der Untersuchungszeitraum von 2000-2005 zu gleichen Teilen repräsentiert wird:

\begin{tabular}{|c|c|c|c|c|}
\hline Jahr & Act & Hit & Album & Non-Hit \\
\hline 2000 & Melanie C. & »| Turn To You« & Northern Star & »Why« \\
\hline 2001 & Enrique Iglesias & $»$ Hero « & Escape & »Love 4 Fun« \\
\hline 2002 & Shakira & »Whenever, Wherever « & Laundry Service & »Fool« \\
\hline 2003 & Evanescence & »Bring Me To Life« & Anywhere But Home & $\begin{array}{l}\text { »My Last } \\
\text { Breath }\end{array}$ \\
\hline 2004 & Britney Spears & »Everytime« & In The Zone & $\begin{array}{l}\text { »Breathe On } \\
\text { Me« }\end{array}$ \\
\hline 2005 & Tokio Hotel & »Durch den Monsun« & Schrei & »Unendlichkeit« \\
\hline
\end{tabular}

Tabelle 1: Randomisiertes Sample der Non-Hits

Aufgrund der verhältnismäßig geringen Anzahl an Non-Hits gegenüber Hits werden die nachfolgenden Vergleiche eher konservativ als Tendenzen bewertet. Dennoch liefern diese Ergebnisse bereits wertvolle Hinweise für

5 Der Hit-Korpus beinhaltet eigentlich 60 Songs mit je zehn Songs pro Jahr - da allerdings drei Songs keine Vers/Chorus-Struktur aufweisen, wurden sie aus dieser Analyse ausgeschlossen. 
potentielle Inkompatibilitäten der Non-Hits mit dem vorliegenden Hitformat, welches sich unter anderem in der Organisation und Gestaltung spezifischer Merkmale ausdrückt.

Die erste Variable erfasst den ersten Einsatzzeitpunkt des ersten Chorus als zentrale Sektion eines Popsongs. Bei Hits liegt der Einsatzzeitpunkt bei einem Mittelwert von 43 Sekunden und einer Standardabweichung von 17 Sekunden (Variable SeC_FCS, siehe Tabelle 2). Hierbei treten weder der Maximalwert von 73 Sekunden noch der Minimalwert von 0 Sekunden statistisch als Ausreißer in Erscheinung. ${ }^{6}$ Diese Verteilung bei Hitsongs deckt sich mit der Einschätzung von Guy Chambers, der als langjähriger Songwriter von Robbie Williams eine Vielzahl von Welthits komponiert hat. In einem BBCInterview bezeichnet Chambers den Einsatzzeitpunkt des ersten Chorus als eine wichtige Regel im Songwriting: »Another big songwriting rule is getting to the chorus before a minute. That is something I am always aware of. I even look at the clock on the computer « (Chambers 2005). Diese Umstände deuten darauf hin, dass es sich bei SeC_FCS um eine Rahmenvariable handelt, die im Kontext des vorliegenden Hitformats einen Wertebereich vorgibt, innerhalb dessen sich eine Merkmalsausprägung bewegen sollte. ${ }^{7}$ Betrachtet man nun die ausgewählten Non-Hits, so zeigt sich, dass zwei Non-Hits, »Why« von Melanie C. und »Breathe On Me« von Britney Spears, mit 110 bzw. 130 Sekunden weit jenseits dieses Rahmenbereichs liegen und somit auf eine Format-Inkompatibilität hindeuten. Überdies zeigt auch der U-Test nach Mann/Whitney einen sehr signifikanten Unterschied zwischen Hits und Non-Hits auf einem Niveau von $p=0.01$ auf (vgl. Tabelle 2$).^{8}$

Das zweite Feature beschreibt die systemische Redundanz der Melodiephrasen im Chorus, indem die gemittelte Selbstähnlichkeit aller Melodiephrasen zueinander berechnet wird. Als Vergleichsmaß wurde hierbei »opti3« implementiert, welches das Ähnlichkeitsurteil von Musikexperten durch die Kombination unterschiedlicher melodischer Ähnlichkeitsmaße mittels einer Regressionsgleichung modelliert (vgl. Müllensiefen 2004: 238ff.).

6 Als Ausreißer werden jene Werte innerhalb eines Boxplots nach Tukey verstanden, die um mehr als das 1,5-fache vom Interquartilabstand entfernt liegen, also des Bereichs, in dem sich die mittleren 50\% aller Werte bewegen (vgl. Bortz 2005: 40; Bühl 2008: 237).

7 Das Attribut Rahmenvariable wird nicht allein aufgrund statistischer Voraussetzungen zugewiesen. Vielmehr spielen hier neben statistischen Gegebenheiten auch musiktheoretische, musikpsychologische als auch kompositionspragmatische Überlegungen eine Rolle. Insofern ist diese Bezeichnung bereits als ein Teil des Interpretationsprozesses zu verstehen.

8 Auf eine ausführliche Beschreibung der am jeweiligen Datenniveau angepassten statistischen Tests wird an dieser Stelle verzichtet und auf die einschlägige Fachliteratur verwiesen, z. B. Bortz 2005. 
Das so entstandene Feature SeMC_RSim_Ch ist auf den Wertebereich 0-1 skaliert, wobei der Wert 1 die sektionsinterne Identität aller Melodiephrasen zueinander beschreibt und 0 deren maximale Unähnlichkeit. Obgleich hier kein signifikanter Unterschied zwischen den beiden Korpora festgestellt werden kann, fällt dennoch der hohe Wert von 0.86 für die systemische Redundanz im Chorus des Tokio Hotel-Titels »Unendlichkeit« auf. In Anbetracht des Umstands, dass sich das Ähnlichkeitsmaß »opti3« sensibel gegenüber leichten Phrasenverlängerungen oder -verkürzungen verhält, deutet dieser hohe Wert auf eine sehr geringe melodische Entwicklung innerhalb dieser Zentralsektion hin. Überdies liegt der Titel von Tokio Hotel sowohl weit über dem Mittelwert von 0.33 (Standardabweichung 0.15) als auch dem Maximum von 0.76 bei Hitsongs. Auf der anderen Seite der Skala liegt bei Non-Hits der Song »Breathe On Me« von Britney Spears mit 0.07 noch unter dem Minimum von Hitsongs, welches bei 0.11 liegt (siehe Tabelle 2). Dieser untere Wert ist allerdings im Anbetracht der zuvor erwähnten Anfälligkeit des Ähnlichkeitsmaßes »opti3《 gegenüber Phrasenverkürzungen und -verlängerungen im Gegensatz zu einem sehr hohen Wert als weniger prekär zu bewerten. Insgesamt weist auch diese Variable vor allem mit ihrem oberen Grenzwert das Potential einer Rahmenvariablen auf, da eine geringe melodische Entwicklung im Chorus nur bedingt durch andere Parameter kompensiert werden kann.

\begin{tabular}{|lcc|}
\hline & $\begin{array}{c}\text { SeC_FCS } \\
\text { Zeitpunkt erstes Erklingen } \\
\text { des Chorus in Sekunden }\end{array}$ & $\begin{array}{c}\text { SeMC_Rsim_Ch } \\
\text { Systemische Redundanz der } \\
\text { Melodiephrasen im Chorus }\end{array}$ \\
\hline Hits Mittelwert & 43 & 0.33 \\
Hits Stdabw. & 17 & 0.15 \\
Hits Minimum & 0 & 0.11 \\
Hits Maximum & 73 & 0.76 \\
Non-Hit »Why« & 110 & 0.14 \\
Non-Hit »Love 4 Fun « & 37 & 0.43 \\
Non-Hit »Fool« & 55 & 0.30 \\
Non-Hit »My Last Breath« & 56 & 0.18 \\
Non-Hit »Breathe On Me« & 130 & 0.07 \\
Non-Hit »Unendlichkeit $«$ & 32 & 0.86 \\
\hline Signifikanztest & Mann-Whitney U-Test & Mann-Whitney U-Test \\
& $p=0.010^{*}$ & $\mathrm{p}=0.195$ \\
\hline
\end{tabular}

Tabelle 2: Merkmale SeC_FCS und SeMC_Rsim_Ch. ${ }^{9}$

9 Die zusätzliche Symbolisierung des statistischen Signifikanzniveaus durch Sternchen folgt der gängigen Konvention in den empirischen Wissenschaften: Signifi- 
Nachdem die ersten beiden Variablen sich mit dem Einsatzzeitpunkt und Redundanzverhältnissen im Chorus beschäftigt haben, soll nun eine weitere Variable einen Aspekt der harmonisch-melodischen Strukturierung dieser Sektion behandeln. So erfasst das nächste Feature die Vorkommenshäufigkeit dreier prominenter kompositorischer Strukturprinzipien, nachfolgend KSPs genannt, innerhalb des Chorus. Sie nehmen eine besonders wichtige Ordnungsfunktion unter den harmonisch-melodischen Variations- und Wiederholungstechniken ein und dienen häufig als organisatorisches Rückgrat des Chorus:

- Melidents - identische Wiederholung einer Melodiephrase über einem veränderten harmonischen Hintergrund,

- Sequenzierung - Wiederholung einer Tonfolge auf einem anderen Tonhöhenniveau (es wird nicht zwischen realer und tonaler Sequenz unterschieden) und

- Intchanges - Wiederholung einer Melodiephrase bei gleichzeitiger Anpassung des Zieltonbereichs auf einem der Taktschwerpunkte an den veränderten Akkordhintergrund (Hybridform aus Melident und Sequenz).
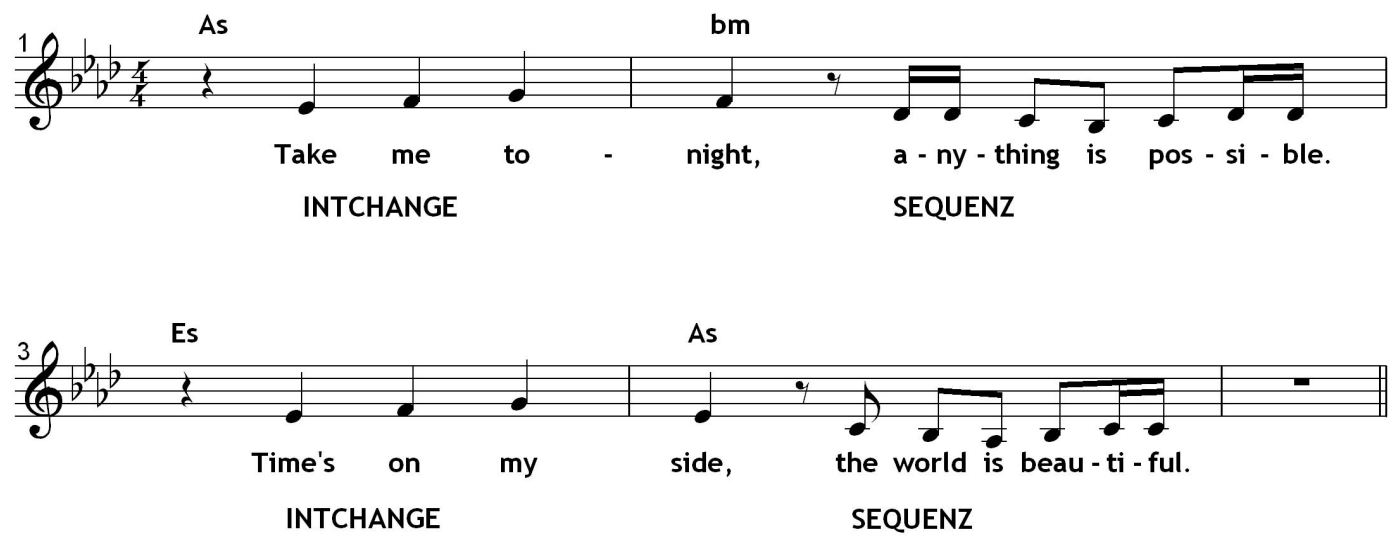

Abbildung 1: Beispiel Sequenzierung und Intchange (Alexander: »Take Me Tonight«)

In Tabelle 3 werden diese drei KSPs zusammengefasst zu der binären Variablen SeMC_KSP_SIM_Ch, welche darüber Auskunft gibt, inwiefern mindestens $50 \%$ aller Melodiephrasen im Chorus von mindestens einem dieser drei Strukturprinzipien organisiert sind. Die oberste und unterste Zeile der Tabelle

kante Aussagen mit einem Sternchen weisen eine Irrtumswahrscheinlichkeit von $p<=0.05$ auf, $p<=0.01$ wird mit zwei Sternchen als sehr signifikant eingestuft und $p<=0.001$ mit drei Sternchen als höchst signifikant (Bühl 2008: 121). Die Signifikanztests beziehen sich hierbei trotz Einzelauflistung der Non-Hits immer auf einen Vergleich der beiden Gesamtkorpora. 
zeigt hierbei an, wieviel Prozent der Hits und Non-Hits das Feature in der Gesamtheit aufweisen - die übrigen Zeilen indizieren mit 0/1 das Fehlen

\begin{tabular}{|c|c|c|}
\hline & $\begin{array}{c}\text { SeMc_KSP_SIM_Ch } \\
\text { Chorusse mit mehr als 50\% } \\
\text { Melidents, Intchanges } \\
\text { und/oder Sequenzierungen } \\
\text { innerhalb der Melodie- } \\
\text { phrasen }\end{array}$ & $\begin{array}{l}\text { SeM_APLD_CPV_3 } \\
\text { Chorusse mit einer } \\
\text { Anhebung des Tonhöhen- } \\
\text { niveaus von mehr als einer } \\
\text { Terz im Vergleich zur } \\
\text { vorhergehenden Sektion } \\
\text { (Vers oder Prechorus) }\end{array}$ \\
\hline Hits & $91 \%$ & $45 \%$ \\
\hline Non-Hit »Why« & 0 & 0 \\
\hline Non-Hit »Love 4 Fun« & 0 & 0 \\
\hline Non-Hit »Fool & 0 & 0 \\
\hline Non-Hit »My Last Breath « & 0 & 0 \\
\hline Non-Hit »Breathe On Me« & 0 & 1 \\
\hline Non-Hit »Unendlichkeit « & 0 & 0 \\
\hline Non-Hits & $0 \%$ & $17 \%$ \\
\hline Signifikanztest & $\begin{array}{l}\text { Fishers Test } \\
p=0.000^{* * *}\end{array}$ & $\begin{array}{l}\text { Fishers Test } \\
p=0.000^{* * *}\end{array}$ \\
\hline
\end{tabular}

Tabelle 3: Binäre Variablen SeMc_KSP_SIM_Ch und SeM_APLD_CPV_3

bzw. Vorkommen des binären Features bei den einzelnen Non-Hits. Der Vergleich zwischen Hits und Non-Hits zeigt, dass sich die beiden Korpora höchst signifikant auf einem Niveau von $p=0.000$ nach Fishers Test unterscheiden. Keiner der Non-Hits weist die zuvor beschriebene mehrheitliche Organisation der Melodiephrasen im Chorus durch diese drei KSPs auf im Gegensatz zu Hitsongs, bei denen eine solche Abdeckung in 91\% aller Songs vorzufinden ist. Die Variable SeMc_KSP_SIM_Ch zeigt eindrucksvoll, dass innerhalb des Formats Hitsong die drei KSPs Melident, Sequenzierung und Intchange als dominierende strukturelle Organisationsprinzipien in Erscheinung treten. Dennoch muss an dieser Stelle angemerkt werden, dass eine derartige Abstraktion nicht die vielfältigen syntaktischen Beziehungen von KSPs abzubilden vermag. So sind weitere kompositorische Strukturprinzipien innerhalb von Hitsongs für die harmonisch-melodische Organisation verantwortlich, wodurch sich eine komplexe Syntax sowohl in vertikaler als auch horizontaler Dimension ausbildet. Diese zeigt sich erst bei einer Verringerung des Abstraktionsniveaus, wodurch in der Folge eine Vielzahl möglicher HitMatrizen zutage treten (Riedemann, in Vorb.). Betrachtet man allein die Abdeckung von $91 \%$ innerhalb der Hitsongs bei dieser Variablen, so kann den drei vorgestellten KSPs jedoch eine hohe Prominenz in dieser Gruppe 
zugesprochen werden. Dennoch eignet sie sich nur bedingt als Rahmenvariable, da offenbar alternative Gestaltungsmittel den Einsatz dieser drei KSPs im Chorus erübrigen können.

Ein in der Hitsongwritingliteratur häufig beschriebenes Hitkriterium stellt die Anhebung des Tonhöhenniveaus zum Chorus dar (vgl. Braheny 2002; Leikin 2008). Dieses Feature wurde nachfolgend so formalisiert, dass sämtliche Tonhöhen der jeweiligen Vorgängersektion zum Chorus (Vers oder Prechorus) unter Berücksichtigung ihrer Dauernwerte gemittelt und anschließend mit dem Tonhöhenniveau des Chorus verglichen wurden. Dabei wurde als Grenzwert einer merklichen Unterscheidung die Anhebung von mehr als einer Terz zugrunde gelegt. Hierbei kann ebenso wie bereits bei der Variablen zuvor ein Unterschied zwischen Hits und Non-Hits auf einem höchst signifikanten Niveau von $p=0,000$ festgestellt werden. So weist nur ein Non-Hit, »Breathe On Me« von Britney Spears, eine deutliche Anhebung des Tonhöhenniveaus zum Chorus auf - im Gegensatz zu Hitsongs, bei denen in $45 \%$ aller Fälle eine solche Anhebung zu registrieren ist.

Ein weiterer wichtiger Analysebereich im Kontext kommerziell erfolgreicher Popmusik stellt die Textebene dar. So bestätigen eine Vielzahl von Untersuchungen die gegenseitige Beeinflussung des Textes auf die Memorabilität von Musik (z.B. Bartlett/Snelus 1980; Crowder et al. 1990; Feierabend et al. 1998), wodurch die textliche Anlage eines Songs einen gewichtigen Einfluss auf die Wahrnehmung des Chorus ausübt. Nachfolgend werden deshalb zwei Variablen vorgestellt, die sich mit der strukturellen Anlage der Songtexte befassen. ${ }^{10}$

Zunächst wird die Dichte syntaktisch-morphologischer Figuren bestimmt, indem die prozentuale Abdeckung der Textphrasen durch Repetitionsformen wie Anaphern als Wiederholung von Satzanfängen, Epiphern als Wiederholung von Satzenden oder Satzwiederholungen und -variationen etc. im Chorus ermittelt wird. Bei der Betrachtung der Ergebnisse der Variablen SeT_SMFD_Ch (Tabelle 4) fällt zunächst der hohe Mittelwert bei Hitsongs auf: Durchschnittlich $82 \%$ aller Textphrasen im Chorus weisen syntaktischmorphologische Figuren bei einer Standardabweichung von 0.17 auf, wodurch sie einen maßgeblichen Einfluss auf die sektionsinterne Strukturierung des Chorus ausüben. Ungeachtet des nichtsignifikanten Ergebnisses im Vergleich zwischen Hits und Non-Hits fällt darüber hinaus auf, dass ein Drittel der Non-Hits überhaupt keine textliche Strukturierung durch diese Gestaltungsmittel aufweisen.

10 Eine weiterführende semantische Analyse der Songtexte findet sich bei Riedemann (in Vorb.); hier wird u.a. die Universalität der Songinhalte auf Grundlage der prominentesten textlichen Propositionen untersucht. 
Aus diesem Grunde soll die Reimdichte als traditionelles formales Stilmittel hinzugezogen werden. Die zweite Variable SeT_SD_Ch (Tabelle 4) stellt ein übergeordnetes Maß zur Beschreibung der textlichen Strukturdichte im Chorus dar. Sie wird gebildet aus dem Mittelwert der zuvor beschriebenen Variablen SeT_SMFD_Ch und der Endreimdichte. Hier weitet sich die zuvor angedeutete Tendenz einer geringeren textlichen Strukturierung bei einigen Non-Hits aus. So bewegen sich die Werte von »Love 4 Fun«, »Fool « und »My Last Breath« deutlich unterhalb des Mittelwert von 0.63 bei Hits und deren Minimum von 0.38. Dieser Unterschied wirkt sich auch auf den Gesamtvergleich zwischen Hits und Non-Hits aus, indem der U-Test nach Mann/Whitney nunmehr einen sehr signifikanten Unterschied auf dem Niveau von $p=0.008$ aufzeigt.

\begin{tabular}{|c|c|c|}
\hline & $\begin{array}{c}\text { SeT_SMFD_Ch } \\
\text { Prozentualer Anteil der } \\
\text { Textphrasen im Chorus mit } \\
\text { syntaktisch-morphologischen } \\
\text { Figuren }\end{array}$ & $\begin{array}{c}\text { SeT_SD_Ch } \\
\text { Gemittelte Strukturdichte } \\
\text { im Chorus unter Berücksich- } \\
\text { tigung von Endreimdichte } \\
\text { und syntaktisch-morphologi- } \\
\text { schen Figuren }\end{array}$ \\
\hline Hits Mittelwert & 0.82 & 0.63 \\
\hline Hits Stdabw. & 0.17 & 0.15 \\
\hline Hits Minimum & 0.5 & 0.38 \\
\hline Hits Maximum & 1 & 1 \\
\hline Non-Hit »Why« & 1 & 0.75 \\
\hline Non-Hit »Love 4 Fun« & 0.5 & 0.25 \\
\hline Non-Hit »Fool « & 0 & 0.2 \\
\hline Non-Hit »My Last Breath « & 0 & 0.2 \\
\hline Non-Hit »Breathe On Me» & 1 & 0.5 \\
\hline Non-Hit »Unendlichkeit« & 1 & 1 \\
\hline Signifikanztest & $\begin{array}{c}\text { Mann-Whitney U-Test } \\
\quad p=0.203\end{array}$ & $\begin{array}{c}\text { Mann-Whitney U-Test } \\
\qquad \mathrm{p}=0.008^{* *}\end{array}$ \\
\hline
\end{tabular}

Tabelle 4: Strukturdichte auf der Textebene

Ungeachtet der Tatsache, dass die vorgestellten Variablen nur einen kleinen Auszug aus der Vielzahl möglicher musik- und textimmanenter Gestaltungsmerkmale darstellen, lässt sich bereits hier mittels einer binär-logistischen Regression die Wahrscheinlichkeit der Zugehörigkeit der Songs zu den Kategorien Hit und Non-Hit in Abhängigkeit dieser Variablen als Einflussgrößen berechnen. Hierbei unterstellt die logistische Regression »einen nicht-linearen Zusammenhang zwischen der Eintrittswahrscheinlichkeit der binären, abhängigen Variablen [...] und den unabhängigen Variablen als Modell- 
prämisse « (Backhaus et al. 2006: 432; Kursivsetzung im Original). ${ }^{11}$ Wie das Ergebnis in Tabelle 5 zeigt, konnten die Songs zu 100\% richtig klassifiziert werden. Als unabhängige Variablen wurden hierbei die vier Features SeC_FCS, SeMC_KSP_SIM_Ch, SeMC_Rsim_Ch sowie SeT_SD_Ch in das Modell eingerechnet.

\begin{tabular}{|c|c|c|c|c|c|}
\hline & & & & orhergesa & \\
\hline & & & & ר-Hit & $\begin{array}{l}\text { Prozentsatz } \\
\text { der Richtigen }\end{array}$ \\
\hline & Beobachtet & & Hit & Non-Hit & Hit \\
\hline Schritt 1 & Hit / Non-Hit & Hit & 57 & 0 & 100,0 \\
\hline & & Non-Hit & 0 & 6 & 100,0 \\
\hline & Gesamtprozer & & & & 100,0 \\
\hline
\end{tabular}

a Der Trennwert lautet ,500

Tabelle 5: Ergebnis der binär-logistischen Regression

So beeindruckend dieses Ergebnis aufgrund der fehlerfreien Klassifizierung auf den ersten Blick zu sein scheint - es wird nicht der Komplexität musikund textimmanenter Gestaltungsmerkmale von Hitsongs gerecht. Insofern ist zu bezweifeln, dass dieses Modell auch außerhalb des vorliegenden Korpus Non-Hits stets zuverlässig zu erkennen vermag, da eine Vielzahl potentieller Format-Inkompatibilitäten in Betracht kommen. Darüber hinaus belegt eine Studie zur Wahrscheinlichkeit zufälliger Doppelschöpfungen im Popmusikbereich, dass selbst unter Vorgabe eines spezifischen Harmoniepatterns kaum Variantenbildungen zu existenten Hits entstanden sind (Frieler/Riedemann 2011). Dennoch lässt sich an den hier vorgestellten Merkmalen bereits das Potential erkennen, welches der computergestützten Analyse zur Beschreibung formatspezifischer Gestaltungsmerkmalen von Hitsongs innewohnt.

Dieser Beitrag zeigt zunächst, dass die so häufig konstatierte Konformität aktueller Charts im Widerspruch zu ihrer strukturellen Komplexität steht. So spannen zusätzlich zu den hier vorgestellten Variablen eine Vielzahl musikund textimmanenter Merkmale ein komplexes Netzwerk auf, welches sich in Form unterschiedlicher Hitmatrizen niederschlägt (Riedemann, in Vorb.). Computergestützte Ansätze stellen in diesem Zusammenhang ein wichtiges Instrument zur analytischen Durchdringung des Materials dar, deren Ergeb-

11 Aufgrund der Robustheit der logistischen Regression gegenüber der Diskriminanzanalyse im Bezug auf die Modellanforderungen wird diesem Verfahren hier der Vorzug gegeben (vgl. auch Backhaus et al. 2006: 425ff.). 
nisse u.a. im Bereich der »corpus-based-musicology « als Basis zur Modellierung kognitiver Modelle der Musikverarbeitung genutzt werden können (Müllensiefen et al 2008: 138, vgl. auch Huron 2006). Überdies ist, wie bereits in der Einleitung angedeutet, ein Transfer zwischen Musikwissenschaft und Musikpraxis denkbar: Erkenntnisse über statistische Verteilungen spezifischer Gestaltungsmittel sowie das Wissen um die strukturelle Organisation und »funktionierende « Matrizen innerhalb eines Formats könnten in die musiktheoretische und -praktische Ausbildung von Songwritern einfließen und für eine Professionalisierung sorgen, wie sie von Seiten der Musikwirtschaft bereits gefordert wurde (Stein 2007: 136f.). Hierbei ginge ein solcher Wissensvorsprung nicht mit einer ästhetischen Nivellierung populärer Musik und kreativer Prozesse einher, im Gegenteil - durch die Kenntnis alternativer Gestaltungsmittel und auch der Möglichkeit des bewussten Bruchs mit formatspezifischen Konventionen verbreitert sich das kreative Ausdrucksspektrum. Zuvor jedoch bliebe die Frage zu klären, wie der Wissenstransfer zwischen Musikwissenschaft und Musikpraxis organisiert und nachfolgend in eine Didaktik überführt werden könnte. Außer Frage steht dabei, dass eine derartige Vernetzung für beide Seiten neue Perspektiven eröffnet.

\section{Literatur}

Abou-Dakn, Masen (2006). Songtexte schreiben. Handwerk und Dramaturgie. Berlin: Autorenhaus Verlag.

Backhaus, Klaus / Erichson, Bernd / Plinke, Wulff / Weiber, Rolf. (2006). Multivariate Analysemethoden. Eine anwendungsorientierte Einführung. Berlin: Springer (11. Aufl.).

Bartlett, James C. / Snelus, Paul (1980). »Lifespan Memory for Popular Songs.«In: American Journal of Psychology 93:3, S. 551-560.

Bortz, Jürgen (2005). Statistik für Human- und Sozialwissenschaftler. Heidelberg: Springer Medizin Verlag (6. aktualisierte Auft.).

Bühl, Achim (2008). SPSS 16. Einführung in die moderne Datenanalyse. München: Pearson Studium.

Braheny, John (2002). The Craft and Business of Songwriting. Cincinatti: Writer's Digest Books (2. Aufl.).

Chambers, Guy (2005). »One of the Judges on Songwriting. Listen to Guy Chambers Talk about Angels, The Beatles and Burt Bacharach« [Interview], http://www. bbc.co.uk/radio2/soldonsong/whatson/guychambers.shtml (Zugriff 1.5.2011).

Crowder, Robert G. / Serafine, Marie Louise / Repp, Bruno H. (1990). »Physical Interaction and Association by Contiguity in Memory for the Words and Melodies of Songs.«In: Memory \& Cognition 18:5, S. 469-476.

Eerola, Tuomas / Järvinen, Topi / Louhivuori, Jukka / Toiviainen, Petri (2001). »Statistical Features and Perceived Similarity of Folk Melodies. « In: Music Perception 18:3, S. 275-296. 
Elflein, Dietmar (2010). Schwermetallanalysen. Die musikalische Sprache des Heavy Metal (= texte zur populären musik 6). Bielefeld: Transcript.

Fabbri, Franco (2004). »A Theory of Musical Genres: Two Applications « [1982]. In: Popular Music. Critical Concepts in Media and Cultural Studies. Hg. v. Simon Frith (= Popular Music Analysis III). London: Routledge, S. 7-35.

Feierabend, John M. / Saunders, T. Clarc / Holahan, John M. / Getnick, Pamela E. (1998). "Song Recognition among Preschool-Age Children: An Investigation of Words and Music. "In: Journal of Research in Music Education 46:3, S. 351-359.

Frieler, Klaus / Riedemann, Frank (2011). »Is Independent Creation Likely To Happen In Pop Music?« In: Musicae Scientiae 15:1, S. 17-28.

Hirschhorn, Joel (2001). The Complete Idiot's Guide to Songwriting. Indianapolis: Alpha Books.

Huron, David (2006). Sweet Anticipation. Cambridge, MA: MIT Press.

Jesser, Barbara (1991). Interaktive Melodieanalyse: Methodik und Anwendung computergestützter Analyseverfahren in Musikethnologie und Volksliedforschung. Typologische Untersuchung der Balladensammlung des DVA. Bern: Peter Lang.

Kachulis, Jimmy (2003). The Songwriter's Workshop. Melody. Boston: Berklee Press.

Kachulis, Jimmy (2010). »Faculty Biography«, http://www.berklee.edu/faculty/ detail/jimmy-kachulis (Zugriff 1.5.2011).

Kopiez, Reinhard / Müllensiefen, Daniel (2011). "Auf der Suche nach den >Popularitätsfaktoren< in den Song-Melodien des Beatles-Albums Revolver: Eine computergestützte Feature-Analyse. In: Musik und Popularität. Beiträge zu einer Kulturgeschichte zwischen 1500 und heute. Hg. v. Sabine Meine und Nina Noeske. Münster: Waxmann, S. 207-226.

Leikin, Molly-Ann (2008). How to Write a Hit Song. Milwaukee: Hal Leonard (5. überarb. Aufl.).

Middleton, Richard (Hg.) (2000). Reading Pop. Approaches to Textual Analysis in Popular Music. Oxford: Oxford University Press.

Middleton, Richard (2001). »Popular Music, I. Popular Music in the West.«In: The New Grove Dictionary of Music and Musicians. Bd. 20. Hg. v. Stanley Sadie. London: Macmillan, S. 128-153.

Moore, Allan F. (Hg.) (2003). Analyzing Popular Music. Cambridge, MA: Cambridge University Press.

Müllensiefen, Daniel (2004). Variabilität und Konstanz von Melodien in der Erinnerung. Ein Beitrag zur musikpsychologischen Gedächtnisforschung. Dissertation Universität Hamburg.

Müllensiefen, Daniel / Wiggins, Geraint / Lewis, David (2008). »High-Level Feature Descriptors and Corpus-Based Musicology: Techniques for Modelling Music Cognition. « In: Systematic and Comparative Musicology: Concepts, Methods, Findings. Hg. von Albrecht Schneider (= Hamburger Jahrbuch für Musikwissenschaft 24). Frankfurt/M. u.a.: Peter Lang, S.133-155.

Pattison, Pat (1991). Songwriting: Essential Guide to Lyric Form and Structure: Tools and Techniques for Writing Better Lyrics. London: Music Sales Ltd.

Perricone, Jack (2000). Melody in Songwriting. Tools and Techniques for Writing Hit Songs. Boston: Berklee Press.

Redmann, Bernd (2002). Entwurf einer Theorie und Methodologie der Musikanalyse. Laaber: Laaber.

Riedemann, Frank (in Vorbereitung). Hitsongs. Eine computergestützte Analyse musik- und textimmanenter Strukturen. Dissertation Hochschule für Musik und Theater Hamburg. 
Rösing, Helmut (2005). »>Populäre Musik<: Was meint das?« [2001]. In: Das klingt so schön hässlich. Gedanken zum Bezugssystem Musik. Hg. v. Alenka Barber-Kersovan, Kai Lothwesen und Thomas Phleps (= texte zur populären musik 2). Bielefeld: Transcript, S. 125-138.

Rösing, Helmut (2002). »>Popularmusikforschung in Deutschland - von den Anfängen bis zu den 1990er Jahren.« In: Musikwissenschaft und populäre Musik. Versuch einer Bestandsaufnahme. Hg. v. Helmut Rösing, Albrecht Schneider und Martin Pfleiderer (= Hamburger Jahrbuch für Musikwissenschaft 19). Frankfurt/M. u.a.: Peter Lang, S. 13-35.

Schmidt, Andre / Terhag, Jürgen (2010). Songwriting. 40 Wege zum eigenen Song. Mainz: Schott.

Silver, Abner / Bruce, Robert (1939). How to Write And Sell a Song Hit. New York: Prentice Hall.

Stein, Thomas M. (2007). »Wen oder was sucht Deutschland?« In: Pop Insights. Bestandsaufnahmen aktueller Pop- und Medienkultur. Hg. v. Thomas Krettenauer und Michael Ahlers. Bielefeld: Transcript, S. 133-139.

Steinbeck, Wolfram (1982). Struktur und Ähnlichkeit: Methoden automatisierter Melodieanalyse (= Kieler Schriften zur Musikwissenschaft 25). Kassel: Bärenreiter.

Tucker, Susan (2003). The Secrets of Songwriting. Leading Songwriters Reveal How to Find Inspiration and Success. New York: Allworth Press.

Wicke, Peter (2003). »Popmusik in der Analyse.« In: Acta Musicologica 75, S. 107126.

\begin{abstract}
Pop music exhibits a variety of structural features and artistic means which contribute to the degree of chart compatibility of a song. Professional songwriters try to internalize these features in order to increase their range of expressive means by manually analysing hit songs. This paper shows the potential contribution of a computational approach within the field of musicology towards the professionalization of hit songwriting. A comparison between hits and non-hits provides valuable first insights into the organisation of hit songs and illustrates possible synergies between musicology, vocational education and economy.
\end{abstract}

